\title{
РАЗРАБОТКА МЕТОДИКИ ОПРЕДЕЛЕНИЯ ОПТИМАЛЬНОГО ДИАМЕТРА УСТЬЕВОГО ШТУЦЕРА
}

\author{
Азеев Александр Александрович1, \\ 3-a@li.ru
}

\author{
Квеско Наталья Геннадьевна 1 , \\ kveskong@gmail.com
}

\author{
Подоляко Дмитрий Сергеевич², \\ podolyakodimas@mail.ru \\ 1 Сибирский федеральный университет, \\ Россия, 660041, г. Красноярск, пр. Свободный, 82, стр. 6. \\ 2 Национальный исследовательский Томский политехнический университет, \\ Россия, 634050, г. Томск, пр. Ленина, 30.
}

\begin{abstract}
Актуальность. Неэфффективное управление системой добычи углеводородов приводит к возрастанию потерь пластовой энергии во всех элементах этой системы из-за образования песчаных и гидратных пробок, ограничения скоростей перекачиваемого потока и к другим осложнениям. Существующая система добычи требует очень точной настройки диаметра штуцера с применением средств автоматизации. На большинстве месторождений России скважины не оборудованы полноценными средствами телемеханики, что существенно ограничивает возможности их управления и контроля.

Цель: разработка методики определения оптимального диаметра штуцера для автоматического управления потоком рабочего агента на основе теоретических исследований системы «пласт-скважина-штуцер».

Методы: сравнительный анализ, системный подход, математическое моделирование.

Результаты. Проводились исследования математической модели процесса многофазной фильтрации через штуцер и в результате были получены зависимости коэффрициента истечения от диаметра штуцера, дебита жидкости, давления до штуцера и обводненности жидкости. Кроме того, были построены регулировочные кривые, описывающие влияние диаметра штуцера на дебит жидкости, давление до штуцера и обводненность жидкости. Установлено, что диаметр штуцера может выступать в качестве ключевого параметра, в наибольшей степени влияющего на возможность управления скважинной системой под контролем компьютерной программы. При этом коэфффициент истечения может быть использован в работе компьютерной программы в качестве основной целевой фуннкции, обеспечивающей выполнение условий вывода работы скважины на нормальный режим. Предложена методика управления скважинной системой, включающей математическую модель процесса многофразной фильтрации через итуцер и алгоритм работы программы управления, которая обеспечивает повышение оперативности и точности регулировки диаметра дросселирующего устройства в широком временном диапазоне работы скважины и связанных с этим изменений фильтрационных характеристик пласта и нарушений в скважинном оборудовании. Использование разрабатываемой системы управления скважиной позволит оптимизировать ее производительность за счет штуцирования в автоматическом режиме, самонастройки под износ и самоочистки штуцера от парафиновых и гидратных пробок, а также снижения вероятности перемерзания и выноса механических примесей. При работе предлагаемой системы управления скважиной дросселирование перекачиваемого фрлюида должно оптимизировать депрессию на пласт, nовышать период фонтанирования и уменьшать негативное влияния газового фактора.
\end{abstract}

\section{Ключевые слова:}

Штуцер, скважина, пласт, методика оптимизации, автоматизация.

\section{Введение}

На нефтегазовых месторождениях крайнего севера существуют проблемы, связанные с необходимостью разработки мероприятий, направленных на снижение обводненности, концентрации механических примесей и контроль газонасыщенности в скважинной продукции, а также увеличение производительности добывающих объектов месторождения. Решение этих проблем может быть связано с устранением несовершенства имеющейся системы управления добычей углеводородов.

Неэффективное управление системой добычи приводит к возрастанию потерь пластовой энергии во всех элементах этой системы из-за образования песчаных и гидратных пробок, ограничения скоростей перекачиваемого потока и к другим осложнениям. При добыче нефти для регулировки расхода жидко- сти, перекачиваемой через устьевую фонтанную арматуру нефтегазовой скважины, используют штуцерные устройства. Резкое сужение диаметра проходного сечения штуцерного устройства может привести к повышению его гидравлического сопротивления, образованию парафиновых и гидратных пробок в нем и, как следствие, к чрезмерному повышению давления на забое. Это может привести к аварии. Резкое расширение диаметра проходного сечения штуцерного устройства приводит к внутрипластовому разгазированию нефти и снижению фазовой проницаемости скважины. Существующая система добычи требует очень точной настройки диаметра штуцера с применением средств автоматизации. На большинстве месторождений РФ скважины не оборудованы полноценными средствами телемеханики, что существенно ограничивает возможности их управления и контроля. 
Для решения указанных проблем в качестве объекта исследования рассматривается процесс управления скважинной системой. При этом цель исследования связана $\boldsymbol{c}$ разработкой методики выбора оптимального диаметра штуцера для управления потоком перекачиваемого флюида на основе теоретических исследований системы «пласт-скважина-штуцер».

\section{Выявление зависимостей между основными} факторами исследуемого процесса

При определении оптимального технологического режима работы скважины необходимо руководствоваться следующими одним или двумя критериями (далее КОТР): неизменная депрессия на пласт; постоянное давление на забое; неизменные величины дебита и скорости восходящего потока; постоянное давление на устье. При обосновании КОТР в первую очередь определяется целевой фактор или группа факторов для уточнения режима работы скважин.

В пределах скважинной системы, состоящей из трех основных элементов - пласт, скважина, штуцер, можно выделить некую структурную взаимосвязь ее основных факторов в виде схемы, показанной на рис. 1. Для поиска и обоснования значений перечисленных выше факторов системы «пласт-скважинаштуцер» целесообразно использовать два основных источника информации: во-первых, это, результаты измерений, которые были проведены в связи с обоснованием работы скважин для стационарных режимов фильтрации; во-вторых, результаты теоретиче- ских исследований на основе методик, которые включают процессы, существующие в условиях разрушения и деформации пласта, возникновения пробки, перетоков подошвенной воды, коррозии и т. д.

На схеме (рис. 1) диаметр штуцера расположен в центре как ключевой параметр, в наибольшей степени влияющий на возможность управления скважинной системой. При предварительном прогнозировании работы скважины очень трудно подобрать оптимальный диаметр штуцера. Как правило, это связано, например, с тем, что в результате изменения фильтрационно-емкостных свойств из-за разрушения пласта или из-за увеличения площади сечения потока через штуцер в ходе эрозии от воздействия песка и других осложнений все исходные проектные расчеты становятся неточными. Кроме того, в длинных штуцерах сложно правильно учесть эффекты, обусловленные трением. В настоящее время процедура корректировки диаметра штуцера на нефтегазовых месторождениях ведется в большей степени в ручном режиме и в силу ряда причин с запозданием в условиях ограниченной информации, не позволяющей достоверно и точно назвать оптимальное значение этого диаметра. Таким образом, целесообразно применение более оперативной и точной регулировки диаметра штуцера в течение длительного периода времени с учетом изменений - фильтрационных характеристик пласта, нарушений в скважинном оборудовании и самой скважине.

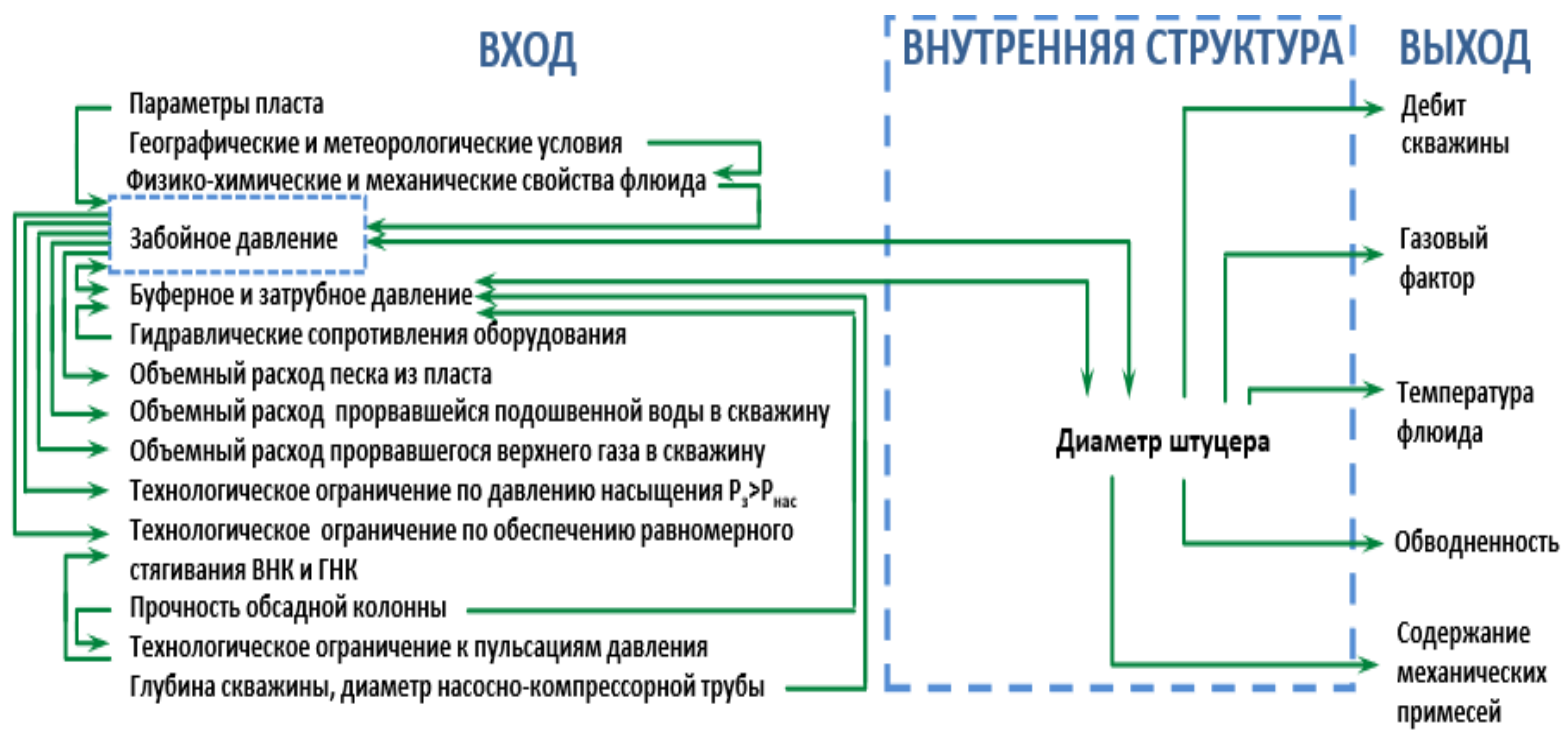

Pис. 1. Схема взаимосвязи основных факторов системы «пласт-скважина-итуцер»

Fig. 1. Diagram of the relationship of the main factors of the "formation-well-nozzle» system

При управлении работой скважины наряду с соблюдением постоянства критерия КОТР важно также контролировать соблюдение следующих условий: максимизировать дебит скважины до значения, не превышающего допустимое; минимизировать содержание механических примесей, обводненность продукции и газовый фактор; исключить рост забойного давления выше допустимого.

\section{Разработка математической модели}

В основе разработки системы штуцерного управления скважиной целесообразно использовать известные зависимости и математические модели многофазной фильтрации через дроссель. В рамках большинства исследований течений многофазных сред через элементы трубопровода, изменяющие сечения потока, пользуются такими понятиями, как 
«критическое» или «звуковое» течение [1]. Например, поверхностные штуцера, как правило, работают в условиях докритического течения и расхода. Сжимаемый многофазный поток в отверстии штуцера может достичь скорости, равной скорости звука. Следовательно, если при течении достигается скорость звука в зоне дросселирования, то теряется зависимость свойства потока от условий, существующих в скважине до штуцера. Соблюдается условие критического течения. Если максимальная скорость потока в зоне дросселирования ниже скорости звука, то такой поток называется докритическим.

Изучение экспериментальных и промысловых данных подтверждает тот факт, что очень сложно получить точный прогноз дебита докритического потока при течении, в частности, через штуцер. Известно [1], что чем меньше разница давлений до и после штуцера, т. е. чем больше диаметр штуцера, тем выше дебит. Когда разница давлений достигает критического значения, расход флюида через штуцер становится постоянным.

При моделировании работы скважинной системы, управляемой штуцером, необходимо учитывать условия перехода критического в докритическое течение, коэффициент критического давления и скорость звука потока.

При расчете скорости многофазного потока по методу Фортунати [2], в качестве допущения, смесь рассматривается как однородная среда при скоростях, не превышающих 10 м/с, с использованием следующей формулы (1)

$$
v_{m 2}=v_{m 2} F\left(\sqrt{\frac{p_{2}}{p_{2} F}}\right)^{\eta},
$$

где $v_{m 2} F$ - реальная скорость смеси до штуцера, м/с; $p_{2}-$ давление до штуцера, кПа, в экспериментах Фортунати принималось равным 1,37 бар; $F$ - коэффициент сопротивления; $\eta$ определяется по формуле (2)

$$
\eta=\left(1-\lambda_{g 2}^{3}\right)^{0,38},
$$

где $\lambda_{\mathrm{g} 2}-$ объемная доля газа после штуцера без учета эффекта проскальзывания, $\mathrm{m}^{3} / \mathrm{m}^{3}$.

Полученное значение скорости необходимо сравнить с данными диаграммы Фортунати [1]. В случае если скорость потока относится к докритической, решение ведется по методике, представленной ниже. В противном случае расчет оптимального диаметра штуцера и других параметров системы необходимо вести с использованием методов Гилберта [3] и Омана [4].

Теоретические зависимости, приведенные далее, разработаны при допущении, что одновременная фильтрация жидкости и газа происходит ниже условий критического расхода (докритический). Изменения в любом дебите или давлении до штуцера не будет, пока не достигнуты границы критической/докритической фильтрации $\left(P_{\text {после штуцера }} \cong 0,5-0,55 P_{\text {до штуцера }}\right)$. На большинстве нефтяных месторождений наиболее популярными являются зависимости, которые также разработаны для многофазной фильтрации через штуцер: зависимость Гилберта [5], Поэтмана и Бэка [6] и Эшфорда [7]. В настоящей работе более подробно рассматривается модель Эшфорда [8].

Ф. Эшфорд разработал модель многофазной фильтрации через штуцер, используя теорию политропного движения. Окончательная форма этого уравнения следующая:

$$
\begin{aligned}
q_{0}= & \frac{1,53 \alpha d^{2} P_{u}}{\left(B_{0}+W O R\right)^{1 / 2}} \times \frac{\left[\left(T_{u} Z_{u}\left(R-R_{s}\right)+151 P_{u}\right)\right]^{1 / 2}}{T_{u} Z_{u}\left(R-R_{s}\right)+111 P_{u}} \times \\
& \times \frac{\left[G_{0}+0,000217 G_{g} R_{s}+(W O R) G_{w}\right]^{1 / 2}}{G_{0}+0,000217 G_{g} R+(W O R) G_{w}},
\end{aligned}
$$

где $q_{0}$ - дебит по нефти, ${ }^{3} /$ ч, в расчетах планируется варьировать в пределах от 0,003 до $0,06 \mathrm{~m}^{3} / \mathrm{c} ; \alpha$ - коэффициент расхода через штуцер; $d$ - диаметр штуцера, м, в расчетах планируется варьировать в пределах от 0,003 до 0,025 м; $P_{u}$ - давление до штуцера, Па; $B_{0}$ - объемный коэффициент нефти, $\mathrm{m}^{3} / \mathrm{m}^{3}$, в расчетах принимается равным 1; WOR - водонефтяное соотношение или водонефтяной фактор, измеряется в долях целого, при $W O R=1$ обводненность составляет $50 \% ; R$ - продуктивное газонефтяное соотношение при стандартных условиях, ст. $\mathrm{M}^{3} /$ ст. $^{3} ; R_{s}$ - растворенное газонефтяное соотношение при штуцерных условиях, ст. $\mathrm{m}^{3} /$ ст. м $^{3} ; T_{u}$ - температура до штуцеpa, ${ }^{\circ} \mathrm{C}$, в расчетах принимается равной $20^{\circ} \mathrm{C} ; Z_{u}-$ коэффициент сжимаемости газа, определенный для условий до штуцера, в расчетах принимается равным $1 ; G_{g}$ - плотность газа, в расчетах принимается равной $0,886 \mathrm{\kappa г} / \mathrm{m}^{3} ; G_{0}$ - плотность нефти, в расчетах принимается равной 0,821 кг/м ${ }^{3} ; G_{w}$ - плотность пластовой воды, в расчетах принимается равной 1160 кг/м ${ }^{3}$. В последующих расчетах забойное давление принимается равным $20 \times 10^{6}$ Па.

Границы корректности изменения перечисленных выше параметров выбраны с учетом работы [9]. Значения для проведения расчетов найдены в ходе анализа и усреднения промысловых данных по нескольким скважинам одного из месторождений Западной Сибири.

Коэффициент сжимаемости газа $Z_{u}$ можно определить по формуле (4) для приближенных расчетов 「10†, при условии изменения приведенных среднекритических значений давления $p_{\text {пр }}$ от 0 до 3 и температуры $T_{\text {пр }}$ от 1,3 до 1,9

$$
\mathrm{Z}_{u}=1-10^{-2}\left(0,76 T_{\text {пр }}^{3}-9,36 T_{\text {пр }}+13\right) \cdot\left(8-p_{\text {пр }}\right) p_{\text {пр }} .
$$

Выражая приведенные среднекритические значения $T_{\text {пр }}$ и $p_{\text {пр }}$ через среднюю относительную плотность газа, получится следующее выражение (5) для определения $Z_{u}$ :

$$
\begin{gathered}
Z_{u}=1-10^{-2}\left(\begin{array}{l}
0,76\left(\frac{T_{\text {раб }}}{171,5 \rho_{\text {г.от }}+97}\right)^{3}- \\
-9,36\left(\frac{T_{\text {раб }}}{171,5 \rho_{\text {г. } . \text { }}+97}\right)+13
\end{array}\right) \times \\
\times\left(8-\left(\frac{p_{\text {раб }}}{\left(4,94-0,46 \rho_{\text {г. } . \text { I }}\right) 10^{6}+97}\right)\right)\left(\frac{p_{\text {раб }}}{\left(4,94-0,46 \rho_{\text {г. } \text { т }}\right) 10^{6}}\right),
\end{gathered}
$$


где $T_{\text {раб }}$ и $p_{\text {раб }}-$ соответственно рабочие давление и температура в Па и К.

Объемный коэффициент и плотность нефти определяются по номограмме Стэндинга [11], кроме того, объемный коэффициент $B_{0}$ можно рассчитать по формуле (6):

$$
B_{0}=1+\lambda_{0} G_{0}+\alpha_{\text {н }}\left(t_{\text {пл }}-20\right)-\beta_{\text {н }} P_{\text {пл }},
$$

где $G_{0}$ - газонасыщенность пластовой нефти, $\mathrm{m}^{3} / \mathrm{m}^{3}$ (объем газа соответствует стандартным условиям); $\beta_{\text {н }}-$ коэффициент сжимаемости дегазированной нефти, $1 / \mathrm{MПа;} P_{\text {пл }}-$ пластовое давление, МПа; $t_{\text {пл }}$ пластовая температура, ${ }^{\circ} \mathrm{C} ; \lambda_{0}-$ эмпирический коэффициент, вычисляемый по формуле (7):

$$
\begin{aligned}
\lambda_{0}=10^{-3}[4,3 & +0,858 \rho_{\text {г }}+5,2\left(1-1,5 \cdot 10^{-3} G_{0}\right) \times \\
& \left.\times 10^{-3} G_{0}-3,54 \bar{\rho}_{\text {нд }}\right],
\end{aligned}
$$

где $\rho_{2}-$ плотность выделившегося газа при $20{ }^{\circ} \mathrm{C}$ и $0,1 \mathrm{MПа,} \mathrm{кг} / \mathrm{M}^{3} ; \rho_{\text {нд }}-$ относительная плотность дегазированной нефти при $20^{\circ} \mathrm{C}$ и $0,1 \mathrm{MПа;} \alpha_{\text {н }}-$ коэффициент термического расширения дегазированной нефти, $1 /{ }^{\circ} \mathrm{C}$, находится с учетом следующего выражения (8):

$$
\alpha_{\text {н }}=10^{-3}\left\{\begin{array}{l}
2,638\left(1,169-\bar{\rho}_{\text {нд }}\right) \\
\text { при } 0,78 \leq \bar{\rho}_{\text {нд }} \leq 0,86 \\
1,975\left(1,272-\bar{\rho}_{\text {нд }}\right) \\
\text { при } 0,86<\bar{\rho}_{\text {нд }} \leq 0,96
\end{array}\right\} .
$$

Водонефтяное соотношение, или водонефтяной фактор WOR, определяется как отношение дебита воды к суммарному дебиту жидкости.

Эшфорд установил, что, если точно рассчитать коэффициент расхода через штуцер $\alpha$ для данной нефтегазовой провинции, то с помощью уравнения (3) можно определить: - дебита, полученного при смене размера штуцера;

- устьевого давления при смене размера штуцера;

- размера штуцера, необходимого для достижения данного устьевого давления и заданного дебита.

Коэффициент расхода через штуцер $\alpha$ определяется по формуле [12]:

$$
\alpha=E C,
$$

где $C$ - коэффициент истечения штуцера [13]; $E$ - коэффициент скорости входа, определяется по формуле

$$
E=1 /\left(1-(d / D)^{4}\right)^{1 / 2},
$$

где $D$ - диаметр трубопровода, м, в расчетах принимается равным 50 мм.

Выражая коэффициент $C$ из формулы (3), с учетом подстановки выражений (9) и (10) для определения $\alpha$ и $E$, получится следующее уравнение:

$$
C=\frac{\left[\begin{array}{l}
q_{0} \sqrt{\left(B_{0}+W O R\right)\left(1-(d / D)^{4}\right)} \times \\
\times\left(T_{u} Z_{u}\left(R-R_{s}\right)+111 P_{u}\right) \times \\
\left.\times\left(G_{0}+0,000217 G_{g} R+(W O R) G_{w}\right)\right]
\end{array}\right.}{\left[\begin{array}{l}
153 d^{2} P_{u} \sqrt{\left[\left(T_{u} Z_{u}\left(R-R_{s}\right)+151 P_{u}\right)\right]} \times \\
\left.\times \sqrt{\left[G_{0}+0,000217 G_{g} R_{s}+(W O R) G_{w}\right]}\right]
\end{array}\right.}
$$

Уравнение (11) является основным уравнением математической модели процесса многофазной фильтрации через штуцер, позволяющим вести расчет основной целевой функции в виде коэффициента истечения, обеспечивающей поиск условий вывода работы скважины на оптимальный режим.

Предполагается, что в случае отклонения какоголибо параметра, учитываемого в уравнении (11), от значений, обеспечивающих оптимальный режим работы скважины, будет корректироваться диаметр штуцера для возврата к оптимальным условиям работы. В этом случае рассчитанное ранее значение коэффициента $C$ подставляется в уравнение, полученное путем выражения диаметра штуцера из формулы (11), которое имеет следующий вид:

$$
d=\sqrt{\left[\begin{array}{l}
q_{0}\left[\left(B_{0}+W O R\right)\left(1 / E^{2}\right)\right]^{\frac{1}{2}} \times \\
\times\left(T_{u} Z_{u}\left(R-R_{s}\right)+111 P_{u}\right) \times \\
\times\left(G_{0}+0,000217 G_{g} R+(W O R) G_{w}\right)
\end{array}\right]}
$$

Формула (12) может использоваться для определения оптимального диаметра штуцера при условии, что коэффициент скорости входа $E$ известен, в противном случае расчет диаметра штуцера ведется по следующей приближенной формуле

$$
d=\frac{1}{2} \sqrt{\frac{2 D-0,211 N^{\frac{1}{3}}+5,8\left(d^{4}-\mathrm{J}\right)}{0,46 N^{\frac{1}{3}}-2,9\left(d^{4}-\mathrm{J}\right)}}-
$$

Расчет коэффициентов $N$ и $J$ ведется с помощью следующих выражений (14), (15)

$$
\begin{gathered}
N=\sqrt{27 D^{4}+256\left(d^{4}-J\right)^{3}}+9 D^{2}, \\
J=\frac{\left[\begin{array}{l}
q_{0}\left(B_{0}+W O R\right)^{\frac{1}{2}}\left(T_{u} Z_{u}\left(R-R_{s}\right)+111 P_{u}\right) \times \\
\times\left(G_{0}+0,000217 G_{g} R+(W O R) G_{w}\right)
\end{array}\right]}{\left[\begin{array}{l}
153 C P_{u}\left[\left(T_{u} Z_{u}\left(R-R_{s}\right)+151 P_{u}\right)\right]^{\frac{1}{2}} \times \\
\times\left[G_{0}+0,000217 G_{g} R_{s}+(W O R) G_{w}\right]^{\frac{1}{2}}
\end{array}\right]} .
\end{gathered}
$$

Формулы (11)-(13) далее планируется взять за основу при разработке алгоритма программы управления скважинной системой.

Так как забойное давление играет немаловажную роль при выборе диаметра штуцера (рис. 1), целесообразно давление до штуцера $P_{u}$ в формулах (11), (12) и (15) выразить через забойное давление. 
В настоящее время известно множество различных подходов к расчету забойного давления, описанных в РД 39-0147035-212-87 [14], в статьях М.М. Хасанова [15], C.S. Kabir [16], A.M. Ansari [17], H.D. Beggs [18], O. Akinsete [19], R.M. El-Saghier [20], M. Firouzi [21], L. Hailong [22], Q. Deng [23], L. Prieto [24], Z. Tariq [25], А.В. Лекомцева [26], В.А. Мордвинова [27], А.В. Лекомцева и В.А. Мордвинова [28], М.С. Турба-

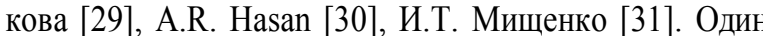
из данных подходов может быть наиболее точным и связанным с разработкой многомерных статистических моделей [32], исходными параметрами для которых являются: динамический уровень, затрубное давление обводненность продукции, дебит жидкости, дебит нефти, глубина скважины до водонефтяного контакта а также, при насосном способе добычи, глубина погружения насоса, в т. ч. под динамический уровень. Многомерные модели, как правило, строятся на основе пошагового регрессионного анализа с использованием метода наименьших квадратов.

Разработка многомерной статистической модели предполагает использование такого количества промысловых данных, которое обеспечит приемлемую точность расчетов. В данном случае при проведении предварительных вычислений важную роль играет в большей степени не точность расчетов, а выяснение характера изменения основных факторов рассматриваемого процесса. Поэтому в рамках настоящей работы при вычислении забойного давления фонтанной скважины предлагается использовать упрощенную методику без привязки к специфике конкретного месторождения, описанную в работе [10].

В ходе решения математической модели на основе формул (11) и (12) следует учитывать следующие допущения [1]: через штуцер протекает адиабатический поток, жидкость является несжимаемой, испарения этой жидкости в штуцере не происходит, многофазная смесь этой жидкости является однородной. Предполагается, что конструкция устьевого штуцера обеспечивает центрирование потока рабочего агента в сочетании с плавной регулировкой диаметра условного прохода. Обобщением полученных выше уравнений служит алгоритм работы программы управления скважинной системой «пласт-скважина-штуцер» (рис. 2), который является важным звеном системы автоматизированного управления работой скважины.

Работа предлагаемой системы управления включает в себя следующую цепочку действий. Вначале вычисляется коэффициент истечения при оптимальных значениях основных факторов скважинной системы по уравнению для определения коэффициент истечения С, а также критерий ОТРС (см. выше). Отклонение исходных факторов системы определяется в ходе постоянного вычисления и сравнения предыдущих и последующих значений коэффициентов истечения. При регистрации отклонения выдается сигнал о корректировке диаметра штуцера на один шаг в сторону уменьшения или увеличения, с учетом того, какой фактор и в какую сторону изменился. Например, увеличение концентрации газовой фазы приводит к падению расхода и давления, и в этом случае целесообразно диаметр штуцера изменять в меньшую сторону.
По окончании первой итерации изменения диаметра штуцера производится вычисление коэффициента истечения и его сравнение с предыдущим значением. Если отклонение находится в допустимых пределах, то диаметр штуцера оставляется без изменений. В противном случае производится дальнейшее изменение диметра штуцера до тех пор, пока значение коэффициента истечения не станет равным исходному при оптимальных значениях основных факторов скважинной системы.

В ходе теоретических исследований математической модели процесса многофазной фильтрации через штуцер предполагается построить регулировочные кривые и проверить, является ли коэффициент истечения той целевой функцией, которая позволит обеспечить выполнение следующих условий вывода работы скважины на нормальный режим:

- увеличение диаметра штуцера при уменьшении дебита;

- увеличение диаметра штуцера при возрастании забойного давления больше критического значения;

- уменьшение диаметра штуцера при увеличении содержания механических примесей;

- уменьшение диаметра штуцера при увеличении обводненности;

- уменьшение диаметра штуцера при увеличении газонасыщенности продукции.

Следует заметить, что положенные в основу разрабатываемой компьютерной программы известные зависимости и математические модели многофазной фильтрации через дроссель не дают убедительных результатов для всего ряда условий эксплуатации, включающих фильтрационные параметры. Следовательно, более-менее законченный вариант используемой в компьютерной программе математической модели процесса может быть получен с учетом результатов анализа промысловых испытаний в ходе отладки и привязки разрабатываемого программного обеспечения применительно к конкретному месторождению.

\section{Анализ математической модели}

Для выяснения характера изменения коэффициента истечения в зависимости от степени влияния таких основных параметров, как диаметр штуцера, дебит жидкости, давление до штуцера, обводненность жидкости, температура потока до штуцера и газонасыщенность, был произведен расчет с использованием уравнения (11).

Известно, что в реальных условиях возможно одновременное изменение почти всех названных выше параметров с разной степенью интенсивности. На текущем этапе исследования для разработки и отладки компьютерной программы управления скважиной вполне достаточно ограничиться одно-, двух факторным экспериментом. В этом случае в ходе расчетов велось изменении каждого вышеперечисленного параметра независимо от остальных, фиксируемых на постоянном уровне. Результаты исследования показаны на рис. 3. 


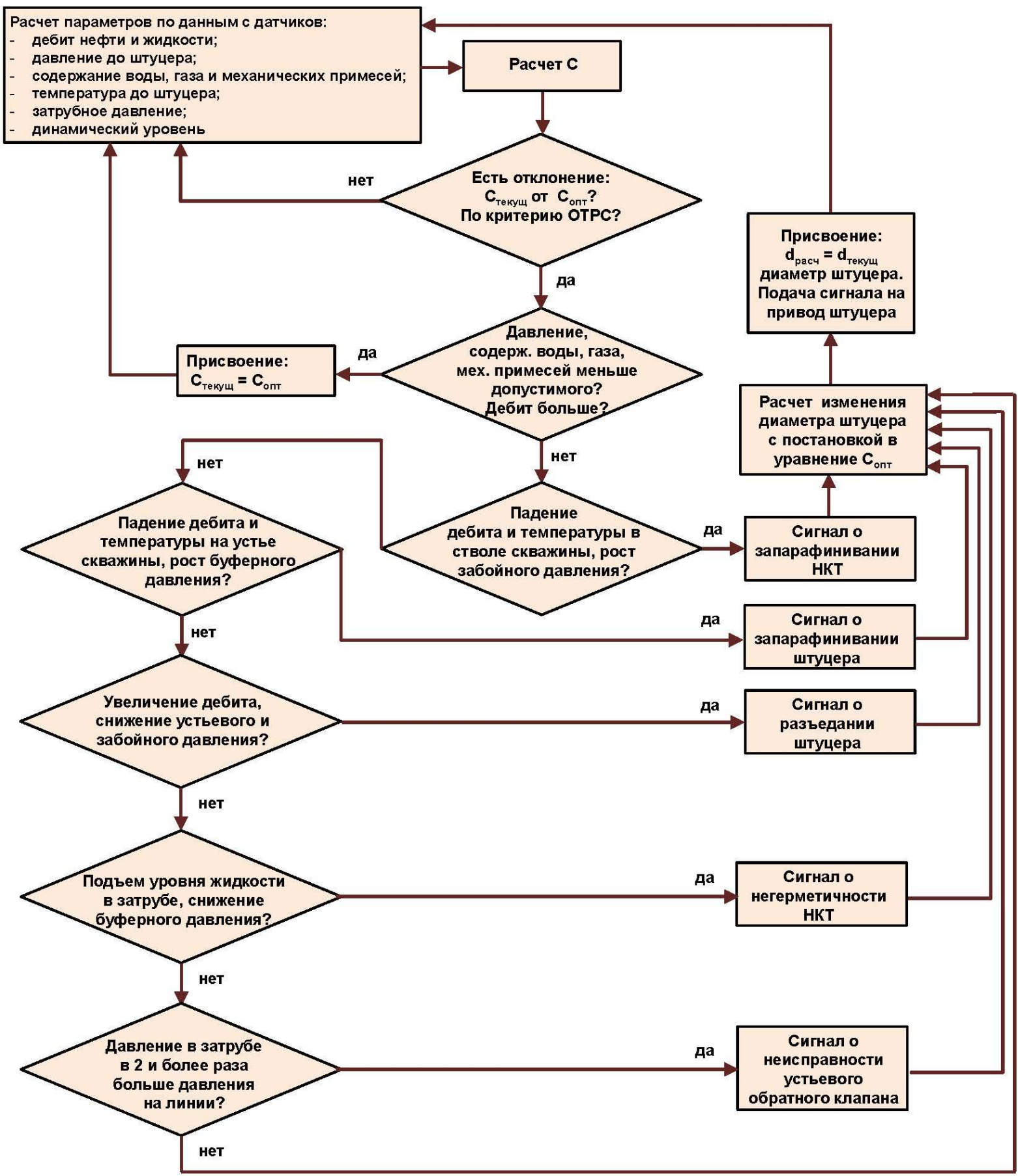

Рис. 2. Алгоритм работы программы управления скважинной системой «пласт-скважина-итуцер»

Fig. 2. Algorithm of operation of the control program of «formation-well-nozzle» well system

Анализ приведенных на рис. 3 графиков показывает, что наиболее заметную степень влияния на коэффициент истечения штуцера оказывает диаметр штуцера в диапазоне от 10 мм и менее, по обратной зависимости. Давление и обводненность также изменяются по обратной зависимости. Прямую зависимость изменения имеют дебит, газонасыщенность и температура до штуцера. При этом анализ уравнения (11) показывает наименьшую степень влияния температуры и газонасыщенности на изменение коэффициента истечения. Наибольшее же влияние оказывает дебит, водонефтяной фактор и, в чуть меньшей, степени давление до штуцера. Примечательно, что диаметр штуцера оказывает наименьшее влияние на значение коэффициента истечения.

В ходе анализа уравнения (12) были построены регулировочные кривые зависимости дебита и давления до штуцера и водонефтяного фактора (обводненности) от диаметра штуцера, приведенные на рис. 4. Кривые изменения температуры и газового фактора на рис. 4 не показаны, так как их изменение было несущественным. 


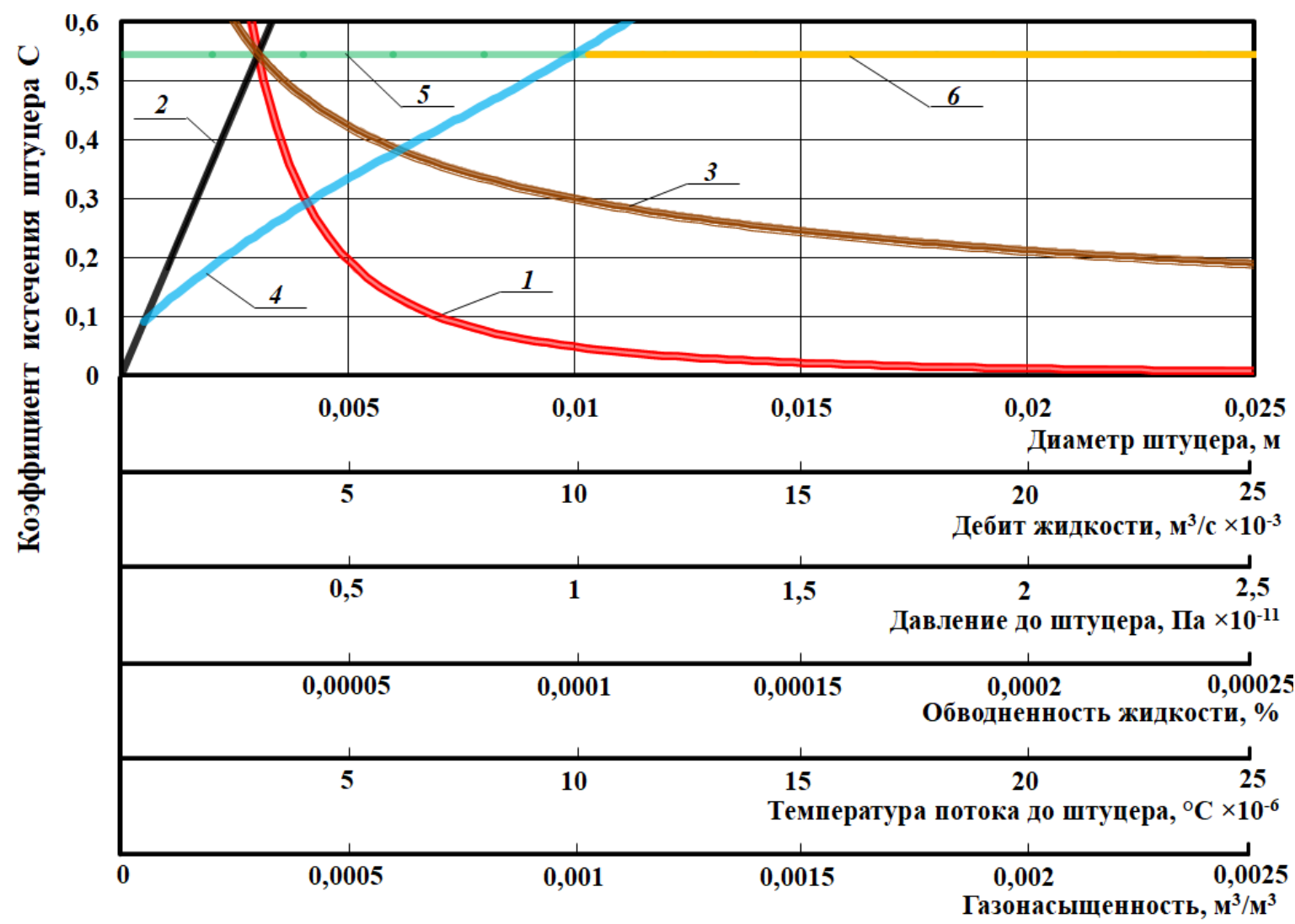

Рис. 3. Зависимости коэффициента истечения итуцера от: 1 - диаметра штуцера, м; 2 - дебита жидкости, $\left(\mathrm{M}^{3} / c\right) \times 10^{-3} ; 3$ - давления до штуиера, Па $\times 10^{-11} ; 4$ - обводненности жидкости, \%; 5 - температуры потока до штуиера, ${ }^{\circ} \mathrm{C} \times 10^{-6} ; 6$ - газонасыщенности, $\mathrm{M}^{3} / \mathrm{M}^{3}$

Fig. 3. Dependences of the nozzle flow coefficient on: 1 - nozzle diameter, $(\mathrm{m}) ; 2$ - liquid flow rate, $\left(\mathrm{m}^{3} / \mathrm{s}\right) \times 10^{-3}$; 3 - pressure up to the nozzle, $\left(\mathrm{Pa} \times 10^{-11}\right) ; 4$ - liquid water cut, \%; 5 -flow temperature up to the nozzle, ${ }^{\circ} \mathrm{C} \times 10^{-6}$; 6- gas saturation, $\mathrm{m}^{3} / \mathrm{m}^{3}$

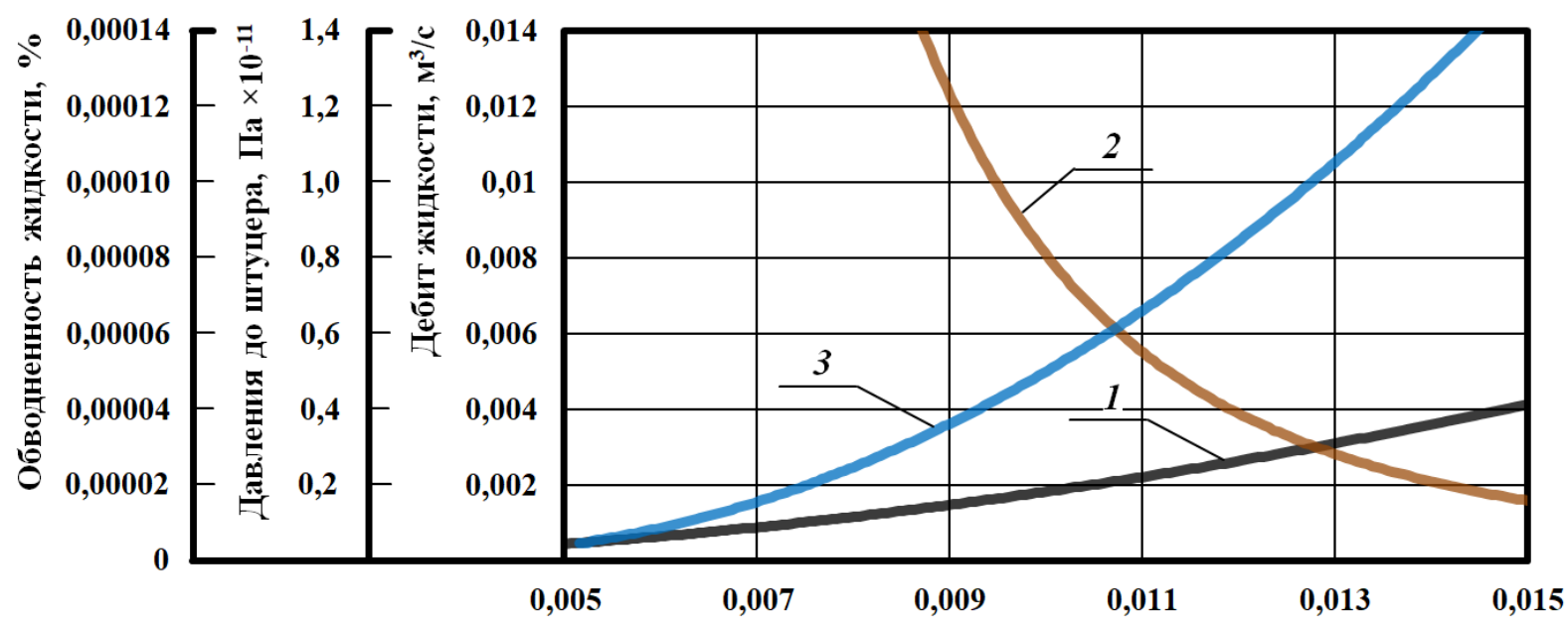

Диаметр штуцера, м

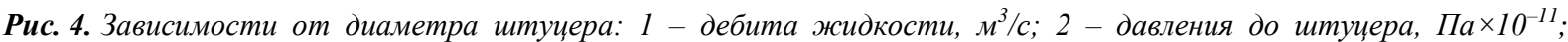
3 - обводненности жидкости, \%

Fig. 4. Dependences of: 1 -liquid flow rate, $\mathrm{m}^{3} / \mathrm{s} ; 2$ - pressure up to the nozzle, $\mathrm{Pa} \times 10^{-11} ; 3$-liquid water cut, \%, on the nozzle diameter

В ходе анализа влияния основных факторов процесса, учитываемых в формуле (12), на изменение диаметра штуцера выяснилось, что в наибольшей степени на изменение диаметра штуцера влияет водонефтяной фактор, затем газовый фактор и темпера- тура и далее по убыванию давление до штуцера, дебит жидкости и коэффициент истечения.

Таким образом, в ходе анализа результатов исследований, приведенных на рис. 3, 4, можно заключить следующее. Не было опровергнуто предположение о 
том, что коэффициент истечения может выступать в качестве основной целевой функции, обеспечивающей выполнение условий вывода работы скважины на нормальный режим. Анализ отклика данной целевой функции на изменение других связанных с ней факторов не выявил противоречия и соответствует известным положениям и законам, описывающим процесс штуцирования многофазного потока. В частности, с увеличением диаметра штуцера дебит и водонефтяной фактор возрастают, а давление до штуцера соответственно уменьшается.

В ходе дальнейшего развития разработанной методики выбора оптимального диаметра штуцера планируется также дополнить существующие математические модели уравнениями, позволяющими вести учет влияния механических примесей на изменение параметров процесса.

\section{Заключение}

В ходе исследования была предложена структурная схема взаимосвязи основных факторов скважинной системы, состоящей из трех основных элементов - пласт, скважина, штуцер. При этом было выявлено, что в качестве ключевого параметра, в наибольшей степени влияющего на возможность управления скважинной системой, может выступать диаметр штуцера. Обеспечение возможности оптимизации диаметра штуцера с учетом основных факторов легло в основу разработки методики управления скважинной системой, включающей математическую модель процесса многофазной фильтрации через штуцер и алгоритм работы программы управления. Планируется в дальнейшем дополнить существующие математические модели уравнениями, позволяющими вести учет влияния механических примесей на изменение параметров процесса.

\section{СПИСОК ЛИТЕРАТУРЫ}

1. Брилл Дж.П., Мукерджи Х. Многофазный поток в скважинах. - М.; Ижевск: Институт компьютерных исследований, 2006. -384 c.

2. Nashawi I., Elgibaly A. Critical two-phase flow through wellhead chokes of middle-east oil wells // IN SITU. - 1997. - № 21. P. 395-427.

3. Ashford F.E. An evaluation of critical multiphase flow performance through wellhead Chockes // JPT. - 1974. - V. 26. № 8. - P. 843-850

4. Nind T.E.W. Principles of oil well production. - New York City: McGraw-Hill Book Company Incorporated, 1964. - 338 p

5. API RP 14J. Design and hazards analysis for offshore production facilities. - Washington: American Petroleum Institute, 2001. - 90 p.

6. ASME B31.4-2008. Pipeline transportation systems for liquid hydrocarbons and other liquids. - New York City: ASME International, 2008. - $125 \mathrm{p}$.

7. ASME B31.8-2020. Gas transmission and distribution piping systems. - New York City: ASME International, 2021. - 224 p.

8. Ashford F.E., Pierce P.E. Determining multiphase pressure drops and flow capacities in down-hole safety valves // JPT. - 1975. V. 27. - № 9. - P. 1145-1152.

9. Оборудование для добычи нефти и газа. Изучение технологий освоения и эксплуатации нефтяных, газовых и нагнетательных скважин / А.А. Азеев, А.В. Матвеев, В.Н. Жигарев, С.В. Лысаков. - Красноярск: Сибирский федеральный университет 2019. $-240 \mathrm{c}$.

10. Юрчук А.М., Истоимн А.3. Расчеты в добыче нефти. - М. Недра, 1979. - 271 с.
Установлена целесообразность работы программного обеспечения в направлении повышения оперативности и точности регулировки диаметра штуцера в широком временном диапазоне работы скважины и связанных с этим изменений фильтрационных характеристик пласта и нарушений в скважинном оборудовании и самой скважине.

При этом компьютерная программа системы управления скважиной должна обеспечивать постоянство критерия оптимального технологического режима, максимизацию дебита в заданных пределах и минимизацию твердой, газовой фазы и содержания воды, а также исключить рост забойного давления выше допустимого.

В результате анализа зависимостей между основными факторами исследуемого процесса добычи углеводородов, полученных в ходе решения математической модели процесса многофазной фильтрации через штуцер, было установлено, что коэффициент истечения может выступать в качестве основной целевой функции, обеспечивающей выполнение условий вывода работы скважины на нормальный режим. Анализ отклика данной целевой функции на изменение других связанных с ней факторов не выявил противоречия и соответствует известным положениям и законам, описывающим процесс штуцирования многофазного потока.

Разработанный алгоритм компьютерной программы направляет управление работой скважины в оптимальную область за счет стабилизации оптимального значения коэффициента истечения штуцера, что обеспечивается в ходе расчета и последующего сравнения предыдущих и последующих значений этого коэффициента, а также принятия решения о подаче сигнала на изменение диаметра штуцера.

11. Standing M.B. Volumetric and phase behavior of oil field hydrocarbon systems. - New York: Reinhold, 1952. - 123 p.

12. Whitfield D.L. Soil farming of oil mud drill cuttings // SPE/IADC Conference. - New Orleans, Louisiana, 1987. - P. 429-438.

13. ГОСТ 8.563.1-97 ГСИ. Измерение расхода и количества жидкостей и газов методом переменного перепада давления. Диафрагмы, сопла ИСА 1932 и трубы Вентури, установленные в заполненных трубопроводах круглого сечения. Технические условия. - М: ИПК Издательство стандартов, 1998. - 64 с

14. РД 39-0147035-212-87. Временное руководство по определению забойного и пластового давления в скважинах механизированного фонда по данным измерений устьевого давления, динамического и статического уровней и давления у приема насоса. - М.: ВНИИнефть, 1987. - 40 с.

15. Мирзаджанзаде А.Х., Хасанов М.М., Бахтизин Р.Н. Моделирование процессов нефтегазодобычи. Нелинейность, неравновесность, неопределенность. - М.; Ижевск: Институт компьютерных исследований, 2004. - 368 с.

16. Hasan A.R., Kabir C.S. Determining bottomhole pressures in pumping wells // SPE J. - 1985. - № 25. - P. 823-838.

17. A Comprehensive mechanistic model for two phase flow in wellbores / A.M. Ansari, N.D. Sylvester, C. Sarica, O. Shoham, J.P. Brill // SPE Production \& Facilities. - 1994. - V. 09. - № 2. - P . 143-152.

18. Beggs H.D., Brill J.P. A study of two phase flow in inclined pipes // Journal of Petroleum Technology. - 1973. - V. 25. № 5. - P. 607-617. 
19. Amar M.N., Zeraibi N., Redouane K. Bottom hole pressure estimation using hybridization neural networks and grey wolves optimization // Petroleum. - 2018. - № 4 (4). - P. 419-429.

20. El-Saghier Rana, Ela Mahmoud, El-Banbi Ahmed. A model for calculating bottom-hole pressure from simple surface data in pumped wells // Journal of Petroleum Exploration and Production Technology. - 2020. - № 10. - P. 2069-2077.

21. Mahshid Firouzi, Suren Rathnayake. Prediction of the flowing bottom-hole pressure using advanced data analytics // SPE/AAPG/SEG Asia Pacific Unconventional Resources Technology Conference. - Brisbane, 2019. - P. 735-742.

22. Liu Hailong. Analysis of factors affecting bottom hole pressure in tight gas reservoir // International Journal of Oil, Gas and Coal Engineering. - 2019. - V. 7. - № 6. - P. 118-124.

23. Numerical investigation of downhole perforation pressure for a deepwater well / Q. Deng, H. Zhang, J. Li, X. Hou, B. Zhao // Energies. - 2019. - V. 12. - P. 1-21.

24. Pressure gradient correlations analysis for liquid-liquid flow in horizontal pipes / L. Prieto, F. Munoz, E. Pereyra, N. Ratkovich // Journal of Petroleum Science and Engineering. - 2018. - № 169. P. 683-704.

25. Tariq Zeeshan, Mahmoud Mohamed, Abdulraheem Abdulazeez. Real-time prognosis of flowing bottom-hole pressure in a vertical well for a multiphase flow using computational intelligence techniques // Journal of Petroleum Exploration and Production Technology. - 2019. - № 10. - P. 1411-1428.
26. Лекомцев А.В., Желанов Е.В., Черных И.А. Статистический подход к оценке забойных давлений в добывающих скважинах // Нефтяное хозяйство. - 2016. - № 10. - С. 98-101.

27. Лекомцев А.В., Мордвинов В.А. К оценке забойных давлений при эксплуатации скважин электроцентробежными насосами // Научные исследования и инновации. - 2011. - Т. 5. № 4. - С. 29-32

28. Лекомцев А.В., Мордвинов В.А. Определение давления у приема электроцентробежных насосов по данным исследований скважин // Вестник ПНИПУ. Геология, нефтегазовое и горное дело. - 2012. - № 4. - С. 84-90.

29. Лекомцев А.В., Мордвинов В.А., Турбаков М.С. Оценка забойных давлений в добывающих скважинах Шершневского месторождения // Нефтяное хозяйство. - 2011. - № 10. C. $30-31$.

30. Hasan A.R., Kabir C.S. Fluid flow and heat transfer in wellbores. - Texas: Society of Petroleum Engineers, 2002. - $181 \mathrm{p}$.

31. Мищенко И.Т. Скважинная добыча нефти. - М.: Нефть и газ, 2003. $-816 \mathrm{c}$.

32. Черных И.А. Определение забойного давления с помощью многомерных статистических моделей (на примере пласта Тл-Бб Юрчукского месторождения) // Вестник ПНИПУ. Геология, нефтегазовое и горное дело. - 2016. - Т. 15. - № 21. - С. 320-328.

Поступила: 26.05 .20212$.

\section{Информация об авторах}

Азеев $\boldsymbol{A . A . , ~ к а н д и д а т ~ т е х н и ч е с к и х ~ н а у к , ~ д о ц е н т ~ к а ф е д р ы ~ р а з р а б о т к и ~ и ~ э к с п л у а т а ц и и ~ н е ф т я н ы х ~ и ~ г а з о в ы х ~ м е - ~}$ сторождений Института нефти и газа Сибирского федерального университета.

Квеско Н.Г., доктор технических наук, заведующая кафедрой разработки и эксплуатации нефтяных и газовых месторождений Института нефти и газа Сибирского федерального университета.

Подоляко Д.С., магистрант Инженерной школы природных ресурсов Национального исследовательского Томского политехнического университета. 
UDC 622.245 .72

\title{
DEVELOPMENT OF PROCEDURE FOR DETERMINING OPTIMAL DIAMETER OF WELLHEAD NOZZLE
}

\author{
Alexander A. Azeev 1 , \\ 3-a@li.ru \\ Nataliya G. Kvesko', \\ kveskong@gmail.com \\ Dmitry S. Podolyako², \\ podolyakodimas@mail.ru \\ 1 Siberian Federal University, \\ 82, Svobodny avenue, Krasnoyarsk, 660041, Russia. \\ 2 National Research Tomsk Polytechnic University, \\ 30, Lenin avenue, Tomsk, 634050, Russia.
}

\begin{abstract}
Relevance. Inefficient management of the production system leads to increased losses of reservoir energy in all elements of this system due to the formation of sand and hydrate plugs, limited rates of pumped flow and other complications. The existing production system requires very precise adjustment of the nozzle diameter using automation tools. In most fields of the Russian Federation, wells are not equipped with full-fledged telemechanics, which significantly limits the ability to manage and control them.

The purpose of the research is to develop a method for determining the optimal diameter of the nozzle for automatic control of the flow of working agent based on theoretical studies of the «formation-well-nozzle» system.

Methods: comparative analysis, system approach, mathematical modeling.

Results. The mathematical model of the multiphase filtration through the nozzle was investigated and as a result, the dependence of the outflow coefficient on the diameter of the nozzle, the fluid flow rate, the pressure to the nozzle and the water content of the liquid was obtained. In addition, the authors have constructed adjustment curves describing the effect of the nozzle diameter on the fluid flow rate, pressure to the nozzle, and liquid watering. It was found that the diameter of the nozzle can act as a key parameter most affecting the ability to control the downhole system with a computer program. The expiration factor can be used in operation of the computer program as the main objective function, which ensures fulfillment of conditions of well operation setting to normal mode. The paper introduces the method of control of well system, which includes mathematical model of multiphase filtration through nozzle and algorithm of operation of control program, which provides for increase of speed and accuracy of adjustment of nozzle diameter during long-term operation of well taking into account the change of filtration pattern of flow in drained volume of formation and disturbances in well itself or equipment installed in it. Use of the developed well control system will allow optimizing its productivity due to automatic assembly, self-adjustment for wear and self-cleaning of the nozzle from paraffin and hydrate plugs, as well as reducing the probability of freezing and removal of mechanical impurities. During the operation of the developed well control system, the flow aggregation should ensure the maintenance of a constant optimal value of depression on the formation, an increase in the period of well runoff and a decrease in the negative effect of the gas factor.
\end{abstract}

\section{Key words:}

Nozzle, well, formation, optimization technique, automation.

\section{References}

1. Brill J.P., Mukherjee H. Mnogofazny potok v skvazhinakh [Multiphase flow in wells]. Moscow, Izhevsk, Computer Research Institute Publ., 2006. 384 p.

2. Nashawi I., Elgibaly A. Critical two-phase flow through wellhead chokes of middle-east oil wells. IN SITU, 1997, no. 21, pp. 395-427.

3. Ashford F.E. An evaluation of critical multiphase flow performance through wellhead chockes. JPT, 1974, vol. 26, no. 8, pp. 843-850.

4. Nind T.E.W. Principles of oil well production. New York City, McGraw-Hill Book Company Incorporated, 1964. 338 p.

5. API RP 14J. Design and hazards analysis for offshore production facilities. Washington, American Petroleum Institute, 2001. $90 \mathrm{p}$.

6. ASME B31.4-2008. Pipeline transportation systems for liquid hydrocarbons and other liquids. New York City, ASME International, 2008. $125 \mathrm{p}$.

7. ASME B31.8-2020. Gas transmission and distribution piping systems. New York City, ASME International, 2021. $224 \mathrm{p}$.

8. Ashford F.E., Pierce P.E. Determining multiphase pressure drops and flow capacities in down-hole safety valves. JPT, 1975, vol. 27, no. 9, pp. 1145-1152.

9. Azeev A.A., Matveev A.V., Zhigarev V.N., Lysakov S.V. Oborudovanie dlya dobychi nefti i gaza. Izuchenie tekhnologiy osvoeniya i ekspluatatsii neftyanykh, gazovykh $i$ nagnetatelnykh skvazhin [Oil and gas production equipment. Study of technologies for development and operation of oil, gas and injection wells]. Krasnoyarsk, Siberian Federal University Publ., 2019. 240 p.

10. Yurchuk A.M., Istoimn A.Z. Raschety $v$ dobyche nefti [Calculations in oil production]. Moscow, Nedra Publ., 1979. $271 \mathrm{p}$.

11. Standing M.B. Volumetric and phase behavior of oil field hydrocarbon systems. New York, Reinhold, 1952. $123 \mathrm{p}$.

12. Whitfield D.L. Soil farming of oil mud drill cuttings. SPE/IADC Conference. New Orleans, Louisiana, 1987. pp. 429-438.

13. GOST 8.563.1-97 GSI. Izmerenie raskhoda $i$ kolichestva zhidkostey i gazov metodom peremennogo perepada davleniya. Diafragmy, sopla ISA 1932 i truby Venturi, ustanovlennye $v$ zapolnennykh truboprovodakh kruglogo secheniya. Tekhnicheskie usloviya [State Standard 8.563.1-97. Measurement of flow rate and quantity of liquids and gases by means of variable pressure drop. Diaphragms, ISA nozzles 1932 and Venturi pipes installed in filled pipelines of round section. Specifications]. Moscow, IPK Standards Publ. House, 1998. $64 \mathrm{p}$.

14. RD 39-0147035-212-87. Vremennoe rukovodstvo po opredeleniyu zaboynogo $i$ plastovogo davleniya $v$ skvazhinakh mekhanizirovannogo fonda po dannym izmereniy ustevogo davleniya, dinamicheskogo $i$ staticheskogo urovney $i$ davleniya $u$ 
priema nasosa [Guidance document 39-0147035-212-87. Temporary manual for determination of bottom hole and formation pressure in wells of mechanized stock based on data of measurements of wellhead pressure, dynamic and static levels and pressure at pump intake]. Moscow, VNIIneft Publ., $1987.40 \mathrm{p}$.

15. Mirzadzhanzade A.H., Khasanov M.M., Bakhtizin R.N. Modelirovanie protsessov neftegazodobychi. Nelineynost, neravnovesnost, neopredelennost [Modeling oil and gas production. Nonlinearity, nonequilibrium, uncertainty]. Moscow, Izhevsk, Computer Research Institute Publ., 2004. 368 p.

16. Hasan A.R., Kabir C.S. Determining bottomhole pressures in pumping wells. SPE J., 1985. no. 25, pp. 823-838.

17. Ansari A.M., Sylvester N.D., Sarica C., Shoham O., Brill J.P. A comprehensive mechanistic model for two phase flow in wellbores. SPE Production \& Facilities, 1994. vol. 09, no. 2, pp. 143-152.

18. Beggs H.D., Brill J.P. A study of two phase flow in inclined pipes. Journal of Petroleum Technology, 1973, vol. 25, no. 5, pp. 607-617.

19. Amar M.N., Zeraibi N., Redouane K. Bottom hole pressure estimation using hybridization neural networks and grey wolves optimization. Petroleum, 2018, no. 4 (4), pp. 419-429.

20. El-Saghier Rana, Ela Mahmoud, El-Banbi Ahmed. A model for calculating bottom-hole pressure from simple surface data in pumped wells. Journal of Petroleum Exploration and Production Technology, 2020, no. 10, pp. 2069-2077.

21. Mahshid Firouzi, Suren Rathnayake. Prediction of the flowing bottom-hole pressure using advanced data analytics. SPE/AAPG/SEG Asia Pacific Unconventional Resources Technology Conference. Brisbane, 2019. pp. 735-742

22. Liu Hailong. Analysis of factors affecting bottom hole pressure in tight gas reservoir. International Journal of Oil, Gas and Coal Engineering, 2019, vol. 7, no. 6, pp. 118-124

23. Deng Q., Zhang H., Li J., Hou X., Zhao B. Numerical investigation of downhole perforation pressure for a deepwater well. Energies, 2019, vol. 12, pp. 1-21.

24. Prieto L., Munoz F., Pereyra E., Ratkovich N. Pressure gradient correlations analysis for liquid-liquid flow in horizontal pipes. Journal of Petroleum Science and Engineering, 2018, no. 169, pp. 683-704.
25. Tariq Zeeshan, Mahmoud Mohamed, Abdulraheem Abdulazeez. Real-time prognosis of flowing bottom-hole pressure in a vertical well for a multiphase flow using computational intelligence techniques. Journal of Petroleum Exploration and Production Technology, 2019, no. 10, pp. 1411-1428.

26. Lecomtsev A.V., Zhelanov E.V., Chernykh I.A. Statisticheskiy podkhod $\mathrm{k}$ otsenke zaboynykh davleniy $\mathrm{v}$ dobyvayushchikh skvazhinakh [Statistical approach to estimation of bottom-hole pressures in producing wells]. Oil industry, 2016, no. 10, pp. 98101.

27. Lekomtsev A.V., Mordvinov V.A. K otsenke zaboynykh davleniy pri ekspluatatsii skvazhin elektrotsentrobezhnymi nasosami [To the assessment of bottom-hole pressures during the operation of wells by electric centrifugal pumps]. Scientific research and innovations, 2011, vol. 5, no. 4, pp. 29-32.

28. Lekomtsev A.V., Mordvinov V.A. Opredelenie davleniya u priema elektrotsentrobezhnykh nasosov po dannym issledovaniy skvazhin [Determination of pressure at the reception of electric centrifugal pumps based on well research data]. Vestnik PNIPU. Geology. Oil and gas and mining, 2012, no. 4, pp. 84-90.

29. Lekomtsev A.V., Mordvinov V.A., Turbakov M.S. Otsenka zaboynykh davleniy v dobyvayushchikh skvazhinakh Shershnevskogo mestorozhdeniya [Assessment of bottomhole pressures in the production wells of the Shershnevskoe field]. Oil industry, 2011, no. 10, pp. 30-31.

30. Hasan A.R., Kabir C.S. Fluid flow and heat transfer in wellbores. Texas, Society of Petroleum Engineers Publ., 2002. 181 p.

31. Mishchenko I.T. Skvazhinnaya dobycha nefti [Downhole oil production]. Moscow, Oil and Gas Publ., 2003. 816 p.

32. Chernykh I.A. Opredelenie zaboynogo davleniya s pomoshchyu mnogomernykh statisticheskikh modeley (na primere plasta $\mathrm{Tl}-\mathrm{Bb}$ Yurchukskogo mestorozhdeniya) [Determination of bottomhole pressure using multidimensional statistical models (using the example of the $\mathrm{Tl}-\mathrm{Bb}$ formation of the Yurchuk deposit)]. Vestnik PNIPU. Geology, oil and gas and mining, 2016, vol. 15, no. 21, pp. $320-328$.

Received: 26 May 2021.

Information about the authors

Alexander A. Azeev, Cand. Sc., associate professor, Siberian Federal University.

Nataliya G. Kvesko, Dr. Sc., head of department, Siberian Federal University.

Dmitry S. Podolyako, graduate student, National Research Tomsk Polytechnic University. 